\title{
Experimental Study on Flexural Fatigue Properties of Reinforced Concrete Beams after Salt Freezing
}

\author{
Jianxi Yang $\mathbb{D}$, ${ }^{1}$ Tianmei Zhang $\left.\mathbb{D}\right)^{1,2}$ and Quansheng Sun $\mathbb{D}^{1}$ \\ ${ }^{1}$ School of Civil Engineering, Northeast Forestry University, Harbin 150040, China \\ ${ }^{2}$ School of Technology, Harbin University, Harbin 150040, China \\ Correspondence should be addressed to Quansheng Sun; hrbsqs@126.com
}

Received 13 August 2019; Revised 22 October 2019; Accepted 14 November 2019; Published 13 January 2020

Academic Editor: Anna Richelli

Copyright (c) 2020 Jianxi Yang et al. This is an open access article distributed under the Creative Commons Attribution License, which permits unrestricted use, distribution, and reproduction in any medium, provided the original work is properly cited.

In order to study the fatigue behavior decay law of reinforced concrete structures in cold region under the action of chlorine salt and freeze-thaw, 150-time water freeze-thaw and salt freeze-thaw cycles of reinforced concrete beams were carried out by the quick freezing method, and then the fatigue properties of the test beams were obtained by the four-point bending fatigue test. The fatigue life of the test beam without freeze-thaw is 1,074,282 times, and the fatigue life of the test beam after freeze-thaw is reduced; the minimum fatigue life of fatigue failure is 493,972 . The test results show that the residual deflection of the test beam is similar to the relative dynamic elastic modulus, which accords with the damage and failure mechanism of concrete, and the growth rate of residual deflection accords with the law of the block model. The fatigue damage model of reinforced concrete specimens is established, the nonlinear fitting of the damage model is carried out according to the test data, the fitting correlation coefficient is more than 0.98 , which indicates that the model can better reflect the damage degree of concrete, and the method of predicting the life of in-service concrete beam is put forward in combination with the concrete damage model.

\section{Introduction}

Reinforced concrete bridges are corroded by salt freezing in the deicing salt environment in cold regions, which leads to concrete erosion and steel corrosion. This not only leads to the decrease of structural bearing capacity but also leads to more serious fatigue damage with repetitive loads such as vehicle loads, wind loads, and wave loads. The fatigue problem greatly reduces the service life of the infrastructure.

1.1. Research Status on Salt-Frost Resistance of Concrete. At present, the durability of concrete under deicing salt conditions is mainly focused on the frost erosion resistance of salt [1-8]. Setzer [9] used RILEM-capillary suction of deicing solution and freeze-thaw test, a concrete salt freezing test method, to compare the surface erosion and internal damage of concrete caused by freeze-thaw cycles. It is concluded that the surface erosion of concrete under salt freezing is dominant, whereas internal damage is more serious under water freeze-thaw. Marchand et al. [10] studied the salt-frost erosion resistance of concrete with different water-cement ratios $(0.25,0.35$, and 0.45$)$ by the ASTMC 672 method. It was considered that the denudation of concrete in $\mathrm{NaCl}$ solution with a concentration of $1.5 \%-3.0 \%$ is the most serious and had nothing to do with water-cement ratio. Foy et al. [11] also used ASTM C 666 and ASTM C 672 methods to study the salt-frost resistance of concrete. It was found that the weight of specimens slightly increased because of capillary water absorption due to cracking of specimens. Janssen's study [12] shows that the weight loss of concrete increases remarkably during the freeze-thaw process of $3.0 \% \mathrm{NaCl}$ solution, but it has little or no effect on the frost resistance index of concrete. The frost resistance index has no relationship with concrete denudation.

1.2. Research Status of Fatigue Behavior of Salt-Frozen Reinforced Concrete Beams. At present, there are many studies on bending fatigue of reinforced concrete beams under cyclic loading only. Fatigue behavior of reinforced 
concrete beams in corrosive environments such as sea water has also been studied [13-15].

The combined action of freeze-thaw and cyclic loads will accelerate the damage of reinforced concrete beams. The research in this field mainly focuses on two aspects. One is to freeze and thaw reinforced concrete beams in water and then to perform the fatigue test to study the fatigue life, crack propagation, fatigue stiffness, and fatigue damage model. Dineshkumar and Ramkumar [16] gave a comprehensive information about the fatigue behavior of reinforced concrete beam with mineral admixtures. The fatigue behavior of reinforced concrete beam depends on the size of the beams, support conditions, number of fatigue cycles, loading limits, and frequency and amplitude of loading applied. Chang and Kesler [17] studied the fatigue behavior of small reinforced concrete beams. The results clearly indicate that the magnitude of repeated load determines the mode of failure. The reinforced concrete beams subjected to fatigue loading fail by the yielding of reinforcement in the tension zone followed by the crushing of concrete in the compression zone [18]. The fatigue life can be calculated from the S-N curve. The $\mathrm{S}-\mathrm{N}$ curve gives the relation between the stress amplitude and the number of fatigue cycles $[19,20]$. Sun et al. [21] paid attention to and studied the deterioration of concrete damage under the coupling action of fatigue load and environmental factors and carried out bending fatigue tests. The dry-wet cycling test of the concrete after fatigue damage was carried out in salt solution, and the chloride ion diffusion resistance of the concrete after fatigue damage was mainly studied.

The other is to consider the corrosion of reinforcement caused by freeze-thaw damage, so the corroded reinforcement is used to manufacture reinforced concrete beams, and the effect of corrosion degree of reinforcement on fatigue performance of reinforced concrete beams is studied. Oyado et al. [22] carried out fatigue tests on reinforced concrete beams with different corrosion rates, and Yi et al. [23] carried out fatigue tests on corroded reinforced concrete beams and analyzed the relationship between the main reinforcement corrosion and the fatigue performance of concrete beams. $\mathrm{Wu}$ et al. [24] studied the failure mode, fatigue strength, fatigue life, and deformation performance of corroded reinforced concrete beams under fatigue load. Li et al. [25] carried out fatigue tests on simulated corroded reinforced concrete beams and analyzed the relationship between main reinforcement corrosion and fatigue life of concrete beams.

It is generally believed that the fatigue durability of structures in the long-term freeze-thaw environment is lower than that of structures in the general atmospheric environment. The coupling effect of the freeze-thaw environment and fatigue load can accelerate the degradation of mechanical properties of structures and components. However, the relevant research is not enough. Especially in the corrosive environment, the fatigue performance of concrete structure after salt-frost damage is still in blank.

At present, there are three kinds of common fatigue life prediction methods: $S-N$ curve method, method based on fatigue cumulative damage theory, and method based on statistical analysis.
Because of its simple form, the $S$ - $N$ curve is suitable for estimating fatigue life under constant amplitude cyclic loading. For the fatigue process under multistage stress and variable amplitude loading, it is impossible to estimate fatigue life directly with the $S$ - $N$ curve [26].

The methods based on fatigue cumulative damage theory belong to deterministic theory, including linear cumulative damage theory [27-31], nonlinear cumulative damage theory [32, 33], and fatigue damage research based on continuum damage mechanics $[34,35]$. The linear cumulative damage theory is widely used in engineering because of its simplicity, convenience, and reliability.

The method based on statistical analysis regards fatigue life $N$ and fatigue damage $D$ as random variables, determines the distribution types of fatigue life and fatigue damage degree, defines the characteristics of critical damage, and uses the statistical analysis method to process these data [36-38].

In this paper, the fatigue behavior of reinforced concrete beams in the deicing salt environment in cold northeast China is studied under salt freezing conditions. Firstly, the rapid freezing method is used to test the fatigue properties of reinforced concrete beams, such as water freezing and salt freezing, respectively, and then four-point bending fatigue tests are carried out. The relative dynamic elastic modulus and residual deflection of reinforced concrete beams under different freezing-thawing media and freezing-thawing cycles are studied and discussed, which can be used for road survey and design departments. In the durability design of concrete structures damaged by salt and frost in cold regions, traffic management departments provide theoretical basis and technical support for the use control of snowmelting materials, the hazard assessment of roads and bridges, and the service life prediction.

\section{Test Situation}

2.1. Specimen Design and Production. The cement adopts Swan brand P.O 42.5 Portland cement produced by Yatai Group. The chemical constituents and percentage composition of cements are shown in Table 1. The compressive strength of the cement test beam is $53.5 \mathrm{MPa}$ in $28 \mathrm{~d}$, and the flexural strength is $8.1 \mathrm{MPa}$ in $28 \mathrm{~d}$. Fine aggregate adopted is river sand with a fineness modulus of 3.36, particle size distribution area 1 . The coarse aggregate used is $5 \sim 20 \mathrm{~mm}$ continuous graded limestone aggregate. Germany BASF polycarboxylic superplasticizer is adopted. The concrete mix ratio of cement: gravel $:$ sand $:$ water $=415: 1219: 600: 166$. The dosage of the water reducer is $0.8 \%$. The average compressive strength of concrete measured by $28 \mathrm{~d}$ cured cube specimens is $f_{\text {cu }}=49.8 \mathrm{MPa}$.

This paper mainly studies the bending fatigue behavior of reinforced concrete beams after freeze-thaw. The size of reinforced concrete beams is limited by the size of the freezethaw test box. The size of reinforced concrete beams is $100 \mathrm{~mm} * 100 \mathrm{~mm} * 400 \mathrm{~mm}$. The reinforcement of the beam is as follows: the main reinforcement and the erection reinforcement are made of two HRB335-class threaded bars with $10 \mathrm{~mm}$ diameter, and the stirrups are made of HPB235- 
TABLE 1: The chemical constituents and percentage composition of cements.

\begin{tabular}{lccccccc}
\hline $\begin{array}{l}\text { Chemical } \\
\text { constituents }\end{array}$ & $\mathrm{SiO}_{2}$ & $\mathrm{Al}_{2} \mathrm{O}_{3}$ & $\mathrm{Fe}_{2} \mathrm{O}_{3}$ & $\mathrm{CaO}$ & $\mathrm{MgO}$ & $\mathrm{SO}_{3}$ & $\mathrm{R}_{2} \mathrm{O}$ \\
\hline $\begin{array}{l}\text { Percentage } \\
\text { composition }\end{array}$ & 21.08 & 5.47 & 3.96 & 62.28 & 1.73 & 2.63 & 0.5 \\
\hline
\end{tabular}

$\mathrm{R}_{2} \mathrm{O}$ refers to other alkali metal oxides.

class round bars with $6 \mathrm{~mm}$ diameter. The mechanical properties of the bars are shown in Table 2. The thickness of concrete cover is $25 \mathrm{~mm}$. The structure and reinforcement diagram of beams are shown in Figure 1. Ten specimens were fabricated, three of which were used for the static load test to determine the ultimate bending load of the test beam. The remaining seven completed the fatigue test after freezing and thawing. The design parameters of the specimens are shown in Table 3.

\subsection{Test Method}

2.2.1. Freeze-Thaw Test. Adopt the "quick freezing method "stipulated in Chinese norms" Test Method for Long-term Performance and Durability of Ordinary Concrete" (GB/T/ 50082-2009) [39]. Freeze-thaw is divided into water freezing and salt freezing (the freeze-thaw medium is $3.5 \% \mathrm{NaCl}$ solution). The specimens were soaked in the corresponding freeze-thaw medium for 4 days and saturated sufficiently. After 28 days of age, the freeze-thaw cycles were carried out 0 times, 50 times, 100 times, and 150 times, respectively. Every 25 freeze-thaw cycles, the mass and relative dynamic modulus of elasticity were measured, then the upper and lower surfaces of the specimen were turned into the specimen box, and the corresponding freeze-thaw medium was replaced.

2.2.2. Fatigue Test. Fatigue tests were carried out on test beams after water freezing-thawing and salt freezingthawing. The test equipment adopts PLS-500 electrohydraulic servo dynamic and static test system, which can automatically control the loading process and automatically collect the test data as shown in Figure 2. The test beam adopts four-point bending fatigue test, as shown in Figure 3. The fatigue load was loaded by the constant amplitude sine wave with a loading frequency of $5 \mathrm{~Hz}$, and stress level is $S=0.65$. The stress level $S$ is the ratio of the maximum fatigue load to the ultimate bearing capacity. The ultimate bearing capacity is obtained by performing a bending test on three unfrozen beams and taking the average of the load at failure. The ultimate load at failure of the three beams is $111.3 \mathrm{kN}$, $98.5 \mathrm{kN}$, and $95.6 \mathrm{kN}$, and the average value is $101.8 \mathrm{kN}$. The maximum fatigue load is $66 \mathrm{kN}$, the minimum fatigue load is $10 \mathrm{kN}$, and the ratio of the lowest load to the highest load is 0.15 .

2.3. Fatigue Test Loading and Data Measurement. In the fatigue test, in order to observe and measure the damage evolution and crack propagation process of the beam, the machine stops and unloads to zero when the number of load cycles reaches $n=0,1 \times 10^{4}, 2 \times 10^{4}, 5 \times 10^{4}, 1 \times 10^{5}, 2 \times 10^{5}$, $5 \times 10^{5}, 1 \times 10^{6}$, and $2 \times 10^{6}$. The maximum residual crack width, dynamic elastic modulus, and maximum residual deflection of the specimen are measured. Then a cyclic static load test is performed. The load is added from zero to the upper limit of fatigue. In this process, the load-deflection curve is measured, the cracks are observed, and the maximum crack width at the upper limit of fatigue is measured.

Residual deflection refers to the maximum deflection of the span measured by unloading the beam after a certain number of load cycles. In order to reduce the test error, two deflectometers are used to measure the midspan deflection of the same specimen at the same time, and the average value is taken as the midspan deflection.

The dynamic elastic modulus is measured by using the dynamic elastic modulus tester as shown in Figure 4. Each test beam is measured twice, and then the average is calculated.

If the number of cycles reaches $n=2 \times 10^{6}$ times and the test beam is still intact, the test will be stopped. According to Chinese standard-Standard for Test Methods of Concrete Structure-if the test beam does not destroy after $2 \times 10^{6}$ cycles, it is considered that it can endure infinite cycles and has infinite life [40].

\section{Test Results and Analysis}

3.1. The Surface Erosion of Freezing-Thawing Specimens. With the increase of freeze-thaw cycles, the surface erosion of salt-frozen specimens is much more serious than that of water-frozen specimens. Figure 5 shows the appearance of the concrete denudation condition on the surface after 150 freeze-thaw tests. It can be seen from Figure 5(a) that the appearance of the water-frozen specimens after 150 times of freezing and thawing is basically intact, only the surface cement slurry drops off, the surface of the test beam is relatively flat, and there is no edge phenomenon of the missing angle. As can be seen from Figure 5(b), the surface of the salt-frozen specimens is severely denuded, the surface of cement mortar is completely exfoliated, and the coarse aggregate is obviously exposed. The middle part of the specimens is uneven, and the edges begin to peel.

3.2. Mass Loss Rate after Freezing and Thawing. As an index of frost resistance, mass loss rate can quantitatively reflect the damage degree of concrete after freeze-thaw erosion. The mass of the test beams with freeze-thaw cycles of 150 times is shown in Table 4 after ever 25 freeze-thaw cycles.

The mass loss rate of the test beam is analyzed and calculated according to the following formula:

$$
\Delta W_{n}=\frac{W_{0}-W_{n}}{W_{0}} \times 100 \%,
$$

where $\Delta W_{n}$ is the mass loss rate of the test beam (\%), $W_{0}$ is the mass of the test beam before freezing-thawing cycle $(\mathrm{kg})$, and $W_{n}$ is the mass of the test beam after $n$ freeze-thaw cycles $(\mathrm{kg})$. 
TABLE 2: Mechanical properties of reinforcing bars.

\begin{tabular}{|c|c|c|c|c|c|}
\hline Strength grade & Steel species & Nominal diameter $(\mathrm{mm})$ & Yield strength $f_{y}(\mathrm{MPa})$ & Ultimate strength $f_{u}(\mathrm{MPa})$ & Elongation $\delta_{10}(\%)$ \\
\hline HRB335 & Ribbed bars & 10 & 335 & 495 & 27 \\
\hline HPB235 & Circular steel bar & 6 & 280 & 419 & 40 \\
\hline
\end{tabular}
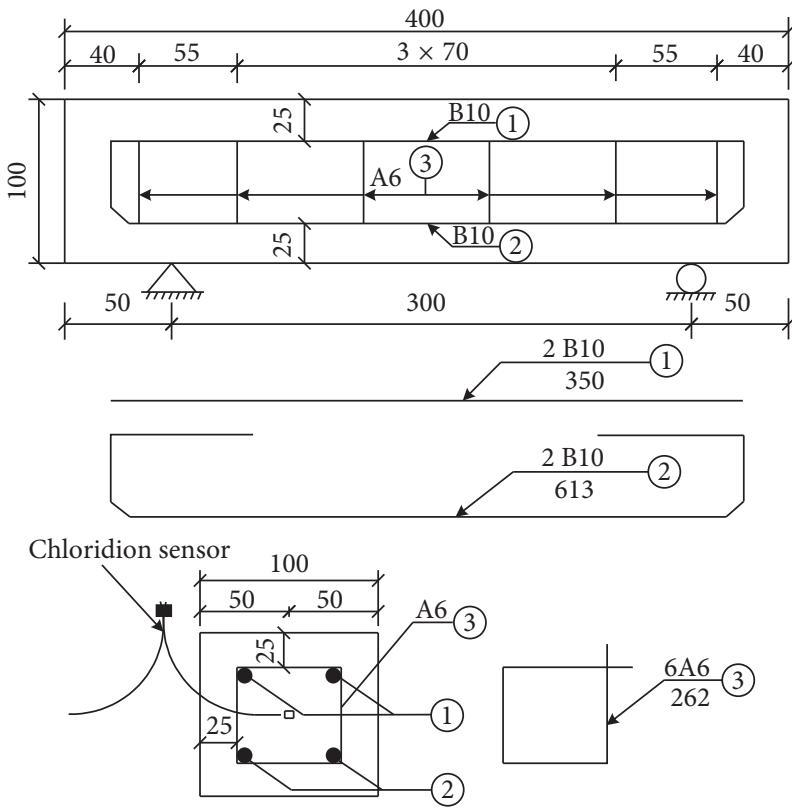

(1) and (2) for rebar reinforcement (3) for round reinforcement

FIGURE 1: Reinforced concrete specimen reinforcement plan (unit: $\mathrm{mm}$ ).

Table 3: Specimen design parameter list.

\begin{tabular}{|c|c|c|}
\hline Test beam number & Freeze-thaw medium & Test situation \\
\hline P1 & - & 0 times freezing and thawing and fatigue test \\
\hline P2 & Water & 50 times freezing and thawing + fatigue test \\
\hline P3 & $\mathrm{NaCl}$ & 50 times freezing and thawing + fatigue test \\
\hline P4 & Water & 100 times freezing and thawing + fatigue test \\
\hline P5 & $\mathrm{NaCl}$ & 100 times freezing and thawing + fatigue test \\
\hline P6 & Water & 150 times freezing and thawing + fatigue test \\
\hline P7 & $\mathrm{NaCl}$ & 150 times freezing and thawing + fatigue test \\
\hline P8, P9, P10 & - & Static load test \\
\hline
\end{tabular}

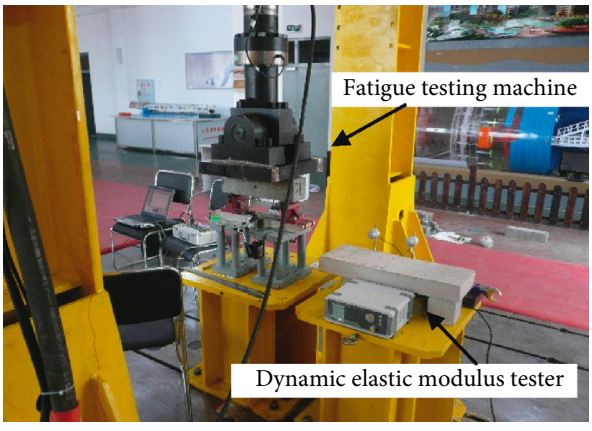

FIgURE 2: Fatigue testing machine.

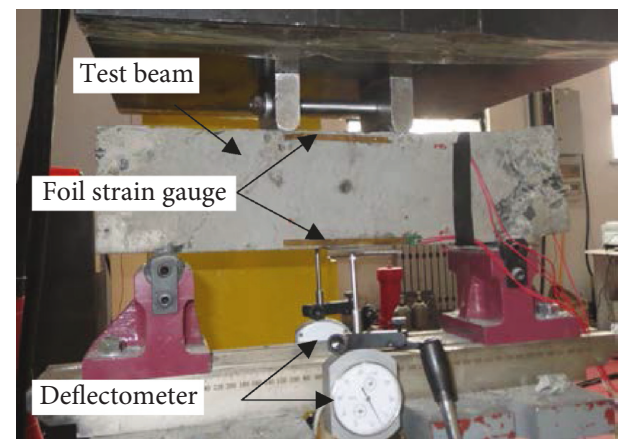

Figure 3: Loading diagram. 


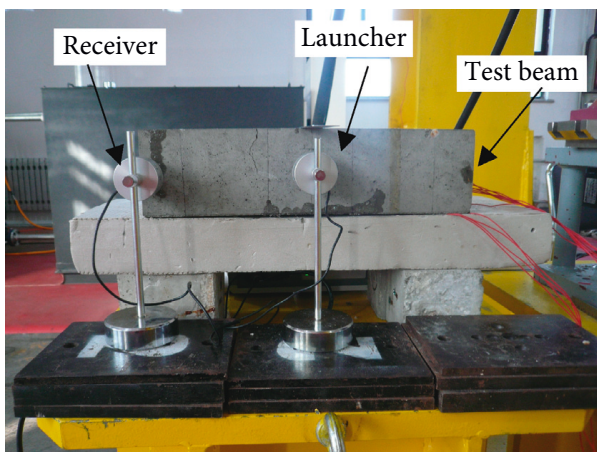

(a)

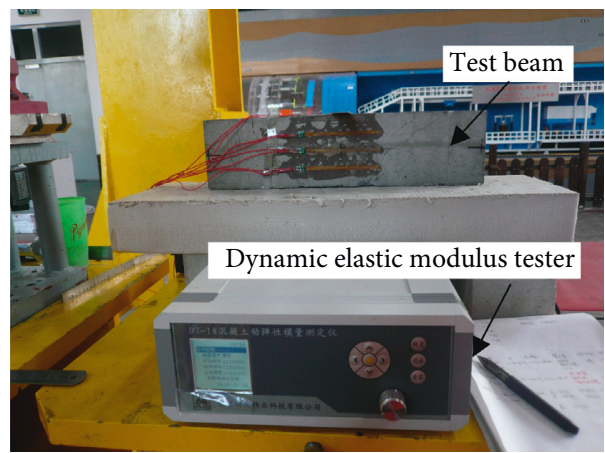

(b)

Figure 4: The dynamic elastic modulus measurement. (a) Front. (b) Back.

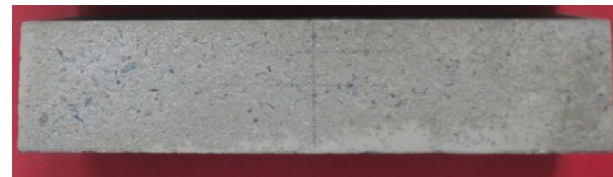

(a)

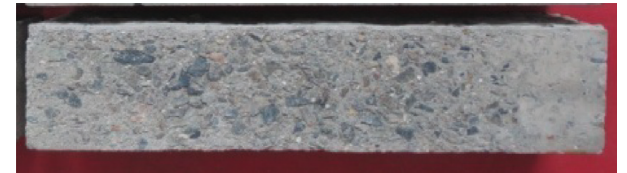

(b)

Figure 5: Test beams after 150 freeze-thaw cycles. (a) Test beam after water freezing. (b) Test beam after $\mathrm{NaCl}$ freezing.

TABle 4: Mass of test beams after freezing-thawing $(\mathrm{kg})$.

\begin{tabular}{lccccccc}
\hline Freezing-thawing cycles & 0 & 25 & 50 & 75 & 100 & 125 & 150 \\
\hline Freezing-thawing in water & 10.495 & 10.506 & 10.517 & 10.544 & 10.441 & 10.437 & 10.443 \\
Freezing-thawing in NaCl & 10.401 & 10.393 & 10.396 & 10.413 & 10.427 & 10.428 & 10.417 \\
\hline
\end{tabular}

Figure 6 is the mass loss rate curve after $0-150$ freezethaw cycles. It can be seen from the graph that the mass loss rate curve of the water-frozen specimen decreases continuously at the beginning of freeze-thaw cycles, indicating that the mass of the specimens increases instead of decreasing; after 125 freeze-thaw cycles, the curve shows an inflection point, the mass loss rate curve shows an upward trend, and water-frozen specimen begins to show mass loss. At the beginning of freezing-thawing (0-50 freezing-thawing cycles), the curve of salt-frozen specimens rises, which indicates that the mass loss occurs, and the change law of the curve is similar to that of the water-frozen specimen.

The reason for the change of mass loss is that the specimen has been immersed in solution for 4 days before the freeze-thaw test and the surface opening pore is saturated with water. At this time, the measured initial mass is the dry mass of the saturated surface. After several freeze-thaw cycles, the water-absorbing saturated opening pore wall is destroyed by the freeze-heave stress cycle. The cement slurry layer on the surface of the specimen peels off quickly. The closed capillary pores which are separated by the airentraining agent bubbles inside the specimen gradually begin to expose and connect. Water then seeps into these micropores and cracks, making the specimen water absorbing and saturated. With the increase of the degree of harmony, the mass of the specimens increases, while the

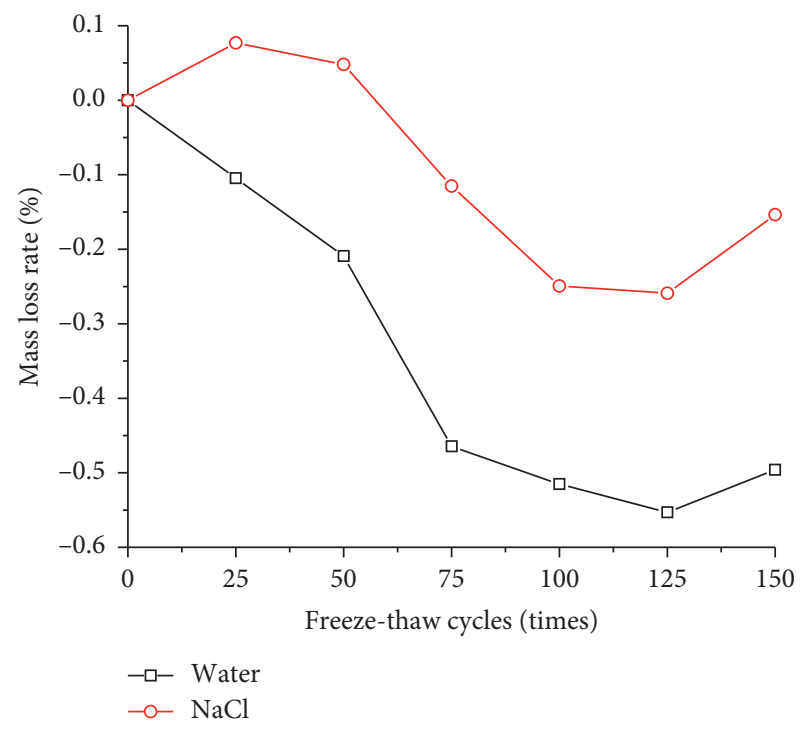

Figure 6: Mass loss rate of the test beam.

mass of concrete decreases due to the erosion of the pore wall caused by frost heave and infiltration. At the beginning of freeze-thaw, the water infiltration is greater than the concrete erosion, so the curve shows a downward trend. With the increase of freeze-thaw cycles, the two are in a 
dynamic equilibrium state. After that, the concrete erosion increases gradually, and the curve shows an upward trend.

In addition to the abovementioned damage, the infiltrated $\mathrm{NaCl}$ reacts with the hydration products of cement, resulting in the over-saturated crystallization of the products. Due to the volume expansion of crystals, the pore wall of concrete is subjected to the action of crystallization pressure as well as hydrostatic pressure and osmotic pressure, and the damage and erosion of pore wall is greater than that of water. On the whole, the overall mass loss of the saltfrozen specimen is greater than that of the water-frozen specimen.

\subsection{Dynamic Elastic Modulus after Freezing and Thawing.} The dynamic elastic modulus can reflect the internal cracks and defects of concrete members and reflect the degree of frost damage of reinforced concrete beams from the microscopic point of view [38]. The dynamic elastic modulus was measured by a dynamic elastic modulus meter and was measured after each 25 freeze-thaw cycles. Each test beam was measured twice and averaged. Table 5 shows the average dynamic elastic modulus of the test beams with 150 times of freeze-thaw cycles.

Figure 7 shows the relative dynamic modulus of elasticity of reinforced concrete beams after $0 \sim 150$ freeze-thaw cycles.

It can be seen from the above results that the relative dynamic elastic modulus decreases with the increase of freeze-thaw cycles. The relative dynamic elastic modulus of water-frozen specimens decreased rapidly than that of saltfrozen specimens. This is because chloride salt reduces the freezing point of frozen water in concrete pores. At the same freezing temperature, the freezing rate in salt-frozen specimens is lower than that in water specimens, which alleviates the internal damage of concrete caused by freezing and thawing, so the relative dynamic elastic modulus decreases slowly. After 150 freeze-thaw cycles, the relative dynamic elastic modulus of the reinforced concrete beams in water and $\mathrm{NaCl}$ solution decreased to $67.4 \%$ and $83.4 \%$ of the initial values, respectively.

3.4. Fatigue Life. Fatigue life is the cycles under fatigue test at failure. After the reinforced concrete test beam is damaged by the freeze-thaw test, the fatigue test is carried out, and the fatigue life decreases to some extent. Table 6 shows the fatigue life of each specimen after different freeze-thaw times.

3.5. Fatigue Failure Mode. There are two typical failure modes of test beams: normal cross section fatigue failure and oblique section fatigue failure, as shown in Figure 8 . No matter what kind of failure mode, the fatigue failure mode of the test beam is sudden fracture. There is no obvious precursor before the failure, and it is brittle fracture. Table 6 shows failure modes of each test beams. According to the failure modes of the beams, there is no clear correlation between the fatigue failure modes and the pre-freeze-thaw medium and the number of freeze-thaw cycles (i.e., freezethaw damage degree). After fatigue failure, the specimens were dissected, and no corrosion marks were found on the surface of steel bars in the specimens.

3.6. Fatigue Residual Crack Width. In the process of fatigue loading, it can be found that the time from loading to macroscopic crack is very short. Generally, macroscopic crack occurs when the fatigue load exceeds the crack load of the beam for the first time, and the main crack occurs near the middle of the span at the earliest. In general, the width of the main crack is the largest and varies with the crack propagation. Figure 9 is the curve of the maximum residual crack width with the fatigue loading times.

From Figure 9, it can be seen that the fatigue residual crack width of both water-frozen specimens and salt-frozen specimens exhibits three stages of development with the number of load cycles: (1) The rapid generation period: at the initial stage of fatigue test, the microcracks visible to the naked eye are rapidly generated, which account for about $0 \% \sim 5 \%$ of the total fatigue life. In this stage, the residual crack width generally does not exceed $0.1 \mathrm{~mm}$, mostly in the range of $0.01-0.1 \mathrm{~mm}$. (2) The slow development stage: this stage accounts for about $90 \%$ of the total fatigue life, the crack width shows very slow growth, the measured residual crack width in this stage is generally in the range of 0.1-0.3 mm, and the crack height almost does not expand. (3) The rapid growth stage: the crack width is about $0.1-0.3 \mathrm{~mm}$. In the last $5 \%$ of the fatigue life, the cracks propagate rapidly and the fatigue failure occurs quickly. The maximum residual crack width is usually $2-4 \mathrm{~mm}$. From the value of residual crack width, the effect of freeze-thaw cycles and freeze-thaw media on the crack width is not obvious.

3.7. Fatigue Dynamic Elastic Modulus. In this paper, when the number of load cycles reaches $n=0,1 \times 10^{4}, 2 \times 10^{4}$, $5 \times 10^{4}, 1 \times 10^{5}, 2 \times 10^{5}, 5 \times 10^{5}, 1 \times 10^{6}$, and $2 \times 10^{6}$ times, downloading is stopped and the dynamic elastic modulus is measured. The curves of the relative dynamic elastic modulus of the test beams after water freezing and salt freezing are shown in Figure 10.

From this diagram, it can be seen that the relative dynamic elastic modulus of the freeze-thaw damaged specimens decreases with the increase of fatigue cycles in the cyclic loading process, and the curves show obvious threestage characteristics: the first stage is about $10 \%$ of the fatigue life, and the relative dynamic elasticity in this stage decreases rapidly. The second stage is about $10 \%-90 \%$ of the fatigue life. In this stage, a platform appears on the graph, and the relative dynamic elastic modulus decreases slowly. The third stage is from $90 \%$ of fatigue life to fatigue failure; when the relative dynamic elastic modulus decreases sharply, the curve shows a significant downward trend. This is consistent with the three-stage characteristics of the crack width development curve.

3.8. Analysis of Variation Law of Fatigue Residual Deflection. Under cyclic loading, the fatigue damage of reinforced concrete beams accumulates continuously. At the beginning 
TABLE 5: Dynamic elastic modulus of the test beam after freezing and melting.

\begin{tabular}{|c|c|c|c|c|c|c|c|}
\hline Freezing-thawing cycles & 0 & 25 & 50 & 75 & 100 & 125 & 150 \\
\hline Freezing-thawing in water & 53.51 & 52.9 & 49.74 & 45.13 & 42.84 & 35.82 & 36.06 \\
\hline Freezing-thawing in $\mathrm{NaCl}$ & 51.49 & 50.95 & 49.67 & 47.45 & 47.74 & 44.2 & 42.96 \\
\hline
\end{tabular}

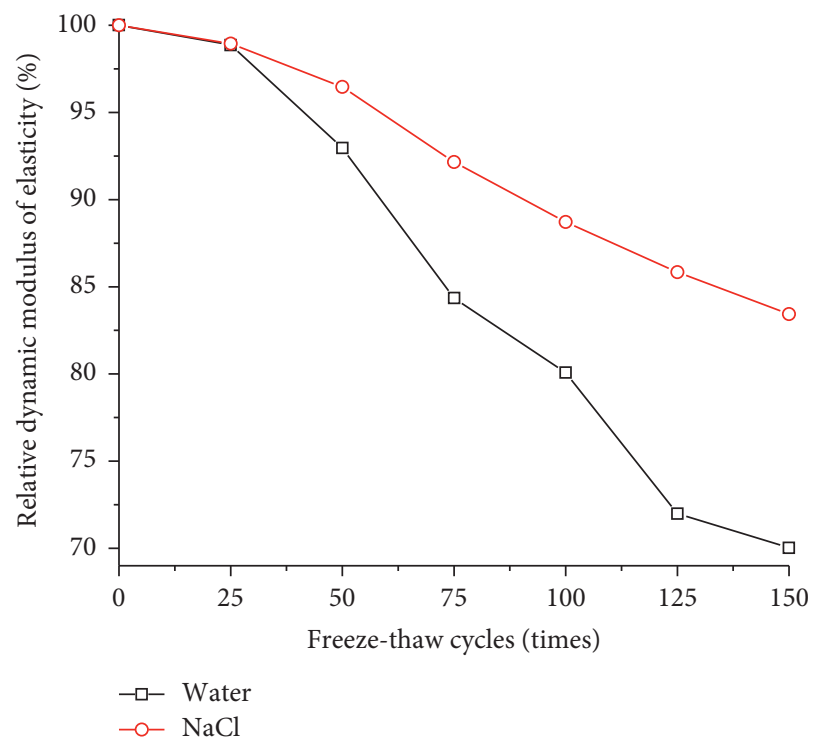

FIGURE 7: Relative dynamic modulus of elasticity.

TABle 6: Fatigue life of test beams.

\begin{tabular}{lcccc}
\hline Test beam number & Freeze-thaw medium & Freezing thawing times & Fatigue life & Fatigue failure mode \\
\hline P1 & - & 0 & 1074282 & Normal cross section failure \\
P2 & Water & 50 & 913734 & Oblique section failure \\
P3 & $\mathrm{NaCl}$ & 50 & 756219 & Normal cross section failure \\
P4 & Water & 100 & 559487 & Oblique section failure \\
P5 & $\mathrm{NaCl}$ & 100 & 701243 & Oblique section failure \\
P6 & Water & 150 & 716758 & Normal cross section failure \\
P7 & $\mathrm{NaCl}$ & 150 & 493972 & Oblique section failure \\
\hline
\end{tabular}

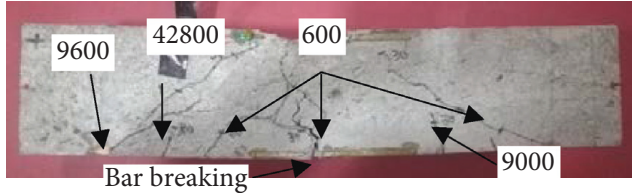

(a)

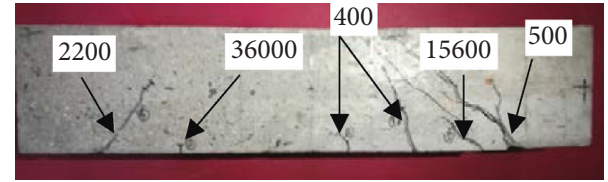

(b)

FIgURE 8: Fatigue failure mode of test beams. (a) Normal cross section failure. (b) Oblique section failure. Number in the fatigue means the number of cycles when each crack started to initiate.

of loading, the concrete in the tension zone cracks, and the tensile steel bars begin to bear more cyclic stress. At the same time, the bond force between the steel bars and the concrete gradually degenerates, the steel bars begin to produce slippage, and the slip displacement increases gradually. As a result, the overall bending stiffness of beams is deteriorated, and the midspan deflection of girders increases continuously. In this paper, the bending stiffness of the beam is reflected by the index of residual deflection. Residual deflection refers to the maximum deflection of the span measured by unloading the beam after a certain number of load cycles. In order to reduce the test error, two deflectometers are used to measure the midspan deflection of the same specimen at the same time, and the average value is 


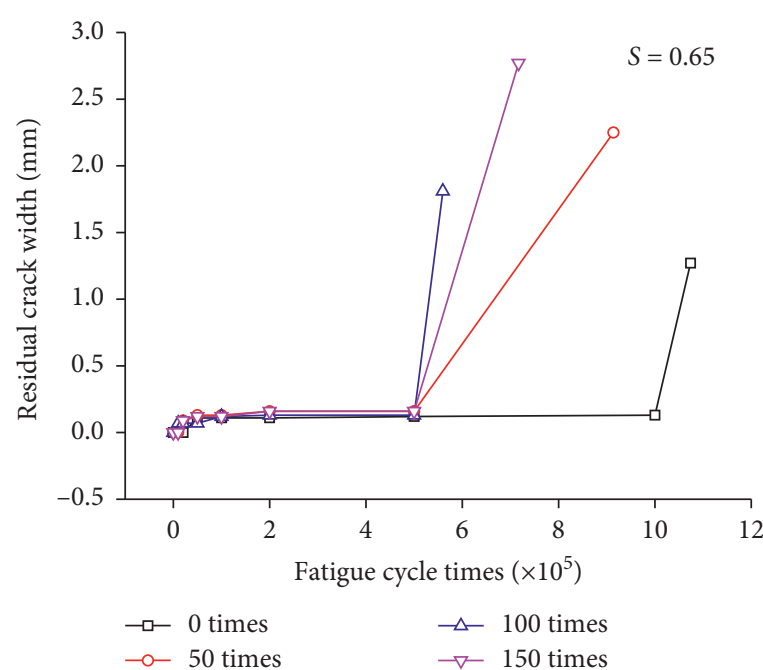

(a)

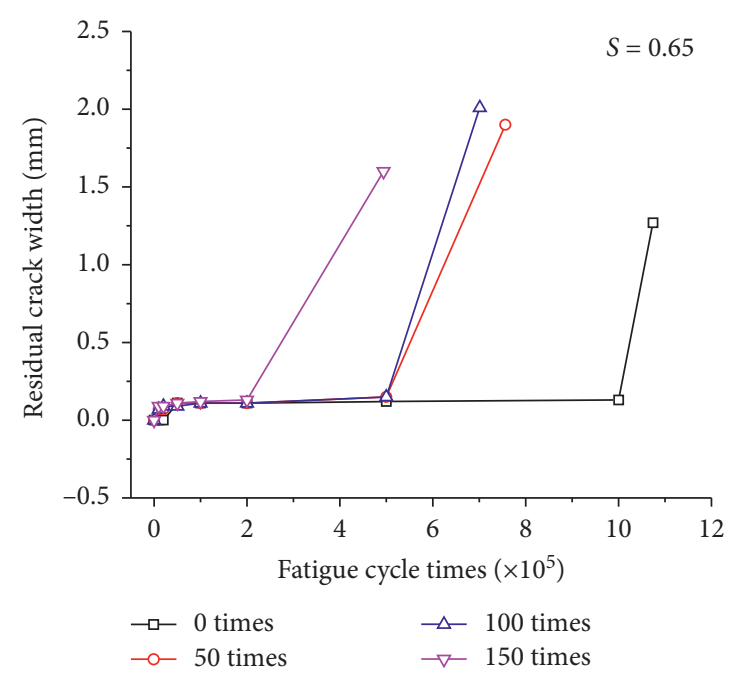

(b)

Figure 9: Residual crack width diagram. (a) Water-frozen specimen. (b) Salt-frozen specimen.

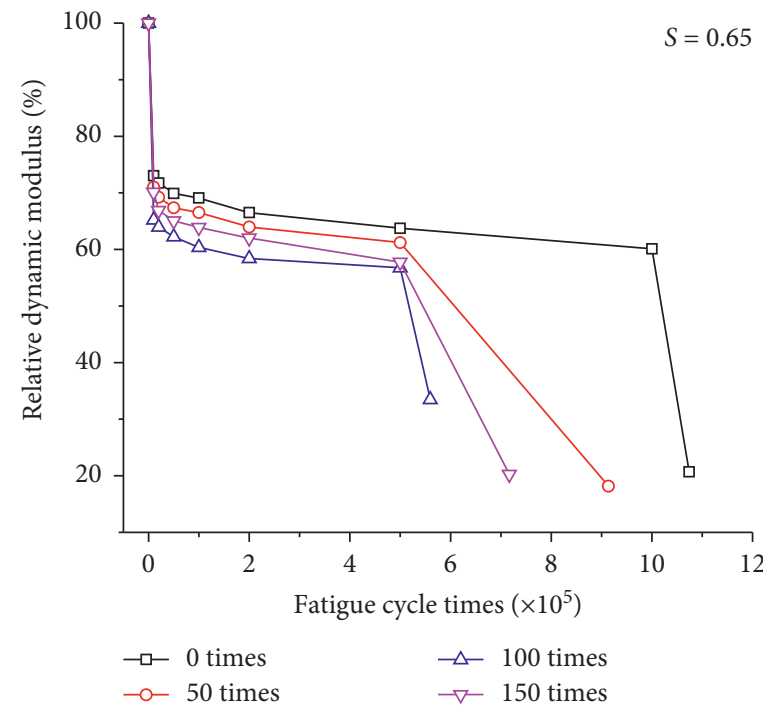

(a)

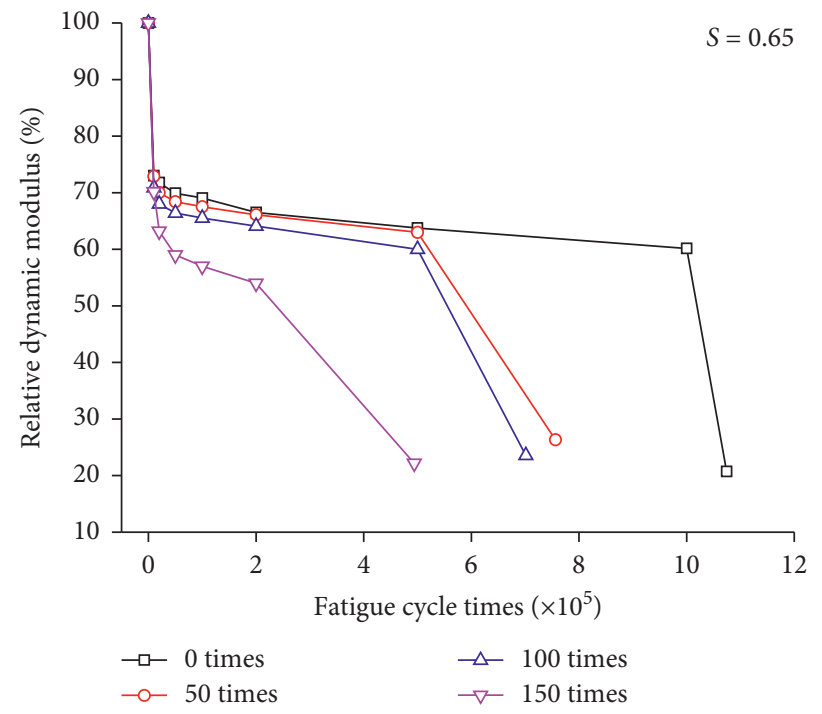

(b)

FIGURE 10: Relative dynamic modulus diagram. (a) Water-frozen specimen. (b) Salt-frozen specimen.

taken as the midspan deflection. The variation of the residual deflection of the water- and salt-frozen specimens with the number of cycles is shown in Figure 11.

It can be seen from Figure 11 that the residual deflection of reinforced concrete specimens after water freezing or salt freezing increases with the increase of load cycles and the stiffness of beams decreases with the increase of load cycles. The variation law of residual deflection is similar to that of the relative dynamic elastic modulus, and it also shows three distinct stages: in the first stage, residual deflection increases rapidly, the second stage curve tends to grow gently, and in the third stage, residual deflection increases rapidly until the fatigue failure.
When the stress level is the same, the residual deflection of the specimens in the same freeze-thaw medium increases with the increase of freeze-thaw cycles, the larger the damage in the early freeze-thaw period, and the residual deflection of the test beam also increases, which is consistent with the results of the dynamic elastic modulus test.

\section{Establishment of Damage Model under Salt Freezing Cycle and Repeated Loading}

4.1. Fatigue Cumulative Damage Model Based on Relative Dynamic Modulus of Elasticity. Because the dynamic elastic modulus is measured by the ultrasonic resonance method, it 


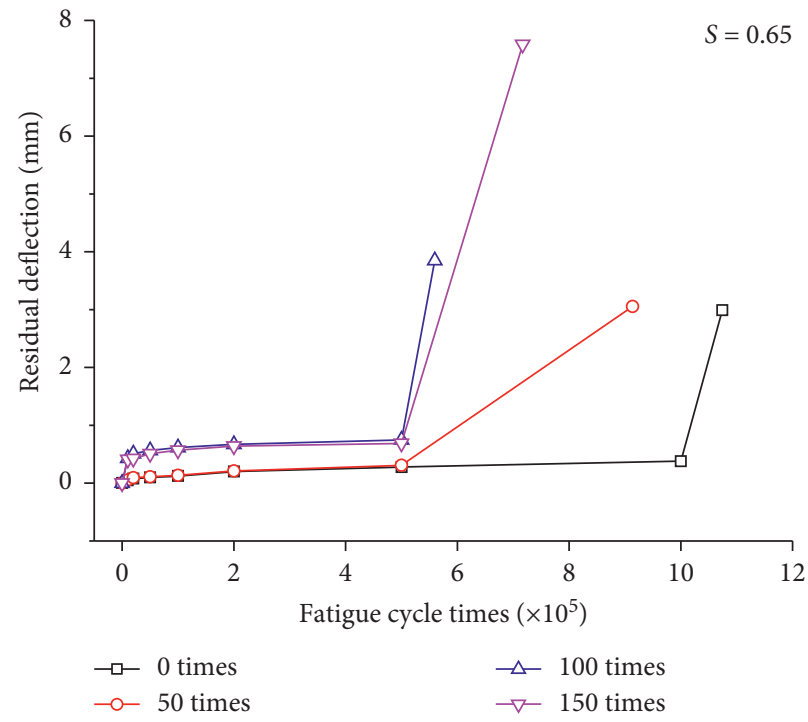

(a)

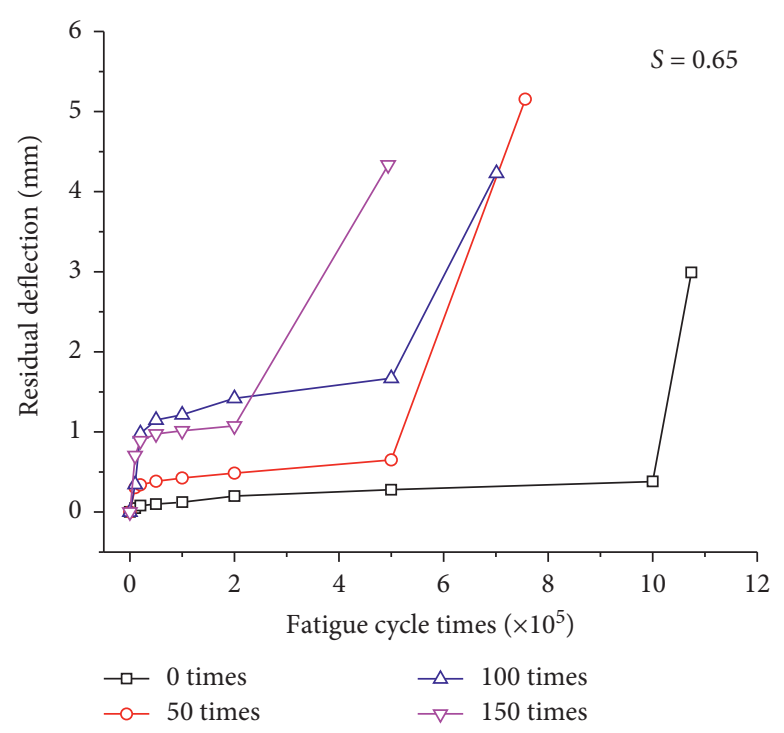

(b)

Figure 11: Residual deflection diagram. (a) Water-frozen specimen. (b) Salt-frozen specimen.

can reflect the change of the internal structure of concrete and the cumulative degree of concrete damage from the microscopic point of view. And the testing speed is fast, and it can realize the nondestructive testing of the structure. The dynamic elastic modulus can be used as the engineering testing means of concrete structure damage. According to the relative Miner criterion [29], the damage degree $D$ can be linearly superimposed by the freeze-thaw damage degree $D_{1}$ and the fatigue damage degree $D_{2}$ :

$$
D=D_{1}+D_{2} \text {, }
$$

where $D$ is the damage degree of the test beam, $D_{1}$ is the damage degree of the test beam due to freeze-thaw cycles, and $D_{2}$ is the damage degree of the test beam due to loading cycles.

According to the theory of damage mechanics, the dynamic elastic modulus is used as a damage factor to define the degree of freeze-thaw damage $D_{1}$ as follows [41]:

$$
D_{1}=1-\frac{E_{n}}{E_{0}}
$$

where $E_{n}$ denotes the dynamic elastic modulus after $n$ freezethaw cycles and $E_{0}$ denotes the initial dynamic elastic modulus before freeze-thaw cycles.

Calculate the freeze-thaw damage degree $D_{1}$ of the test beam in Table 5 according to formula (3), and perform regression analysis to fit the relationship between the freezethaw damage degree $D_{1}$ and freeze-thaw cycles $n$, as shown in Figure 12. The fitted curve conforms to the exponential form and agrees well with the experimental values, and the correlation coefficient is greater than 0.96 . The fitting formula is

$$
D_{1}=1-\frac{E_{n}}{E_{0}}=a_{0}+a_{1} e^{n / t},
$$

where $a_{0}, a_{1}$, and $t$ are the coefficients related to the material, freeze-thaw medium, and freeze-thaw times, which can be obtained from Figure 12, and $n$ is the number of freeze-thaw cycles.

The fatigue damage degree $D_{2}$ is defined as

$$
D_{2}=1-\frac{E_{d N}}{E_{d 0}},
$$

where $E_{d N}$ denotes the fatigue dynamic elastic modulus after $N$ cycles of loading and $E_{d 0}$ denotes the initial fatigue dynamic elastic modulus (i.e., the dynamic elastic modulus after freezing and thawing). The ratio between them is the relative dynamic elastic modulus of fatigue.

From Figure 10, the curves show obvious three-stage characteristics, the second stage is about $10 \%-90 \%$ of the fatigue life, and it is more important, so the first and third stages with less impact could be ignored. In the second stage, the curve of fatigue relative dynamic elastic modulus changing with the number of load cycles $N$ is close to a straight line, and the attenuation model of the relative dynamic elastic modulus is obtained by linear regression:

$$
\frac{E_{d N}}{E_{d 0}}=a_{2}-a_{3} N, \quad\left(0.1 N_{f} \leq N \leq 0.9 N_{f}\right),
$$

where $a_{2}$ is the relative dynamic elastic modulus at the end of the first stage, which is greatly affected by the early freezethaw damage, and $a_{3}$ is the second-stage linear slope, which indicates the relative attenuation rate of fatigue to dynamic elastic modulus and is related to the stress level.

Formulas (4)-(6) are substituted into formula (2) considering initial freeze-thaw damage in the fatigue cumulative damage model can obtain

$$
D=\left(a_{0}+a_{1} e^{n / t}\right)+\left(1-a_{2}+a_{3} N\right),
$$




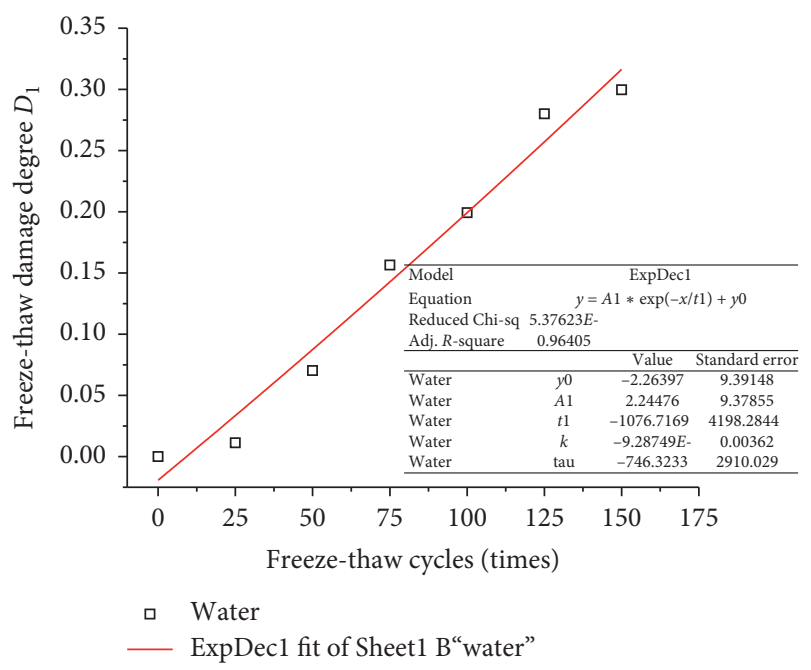

(a)

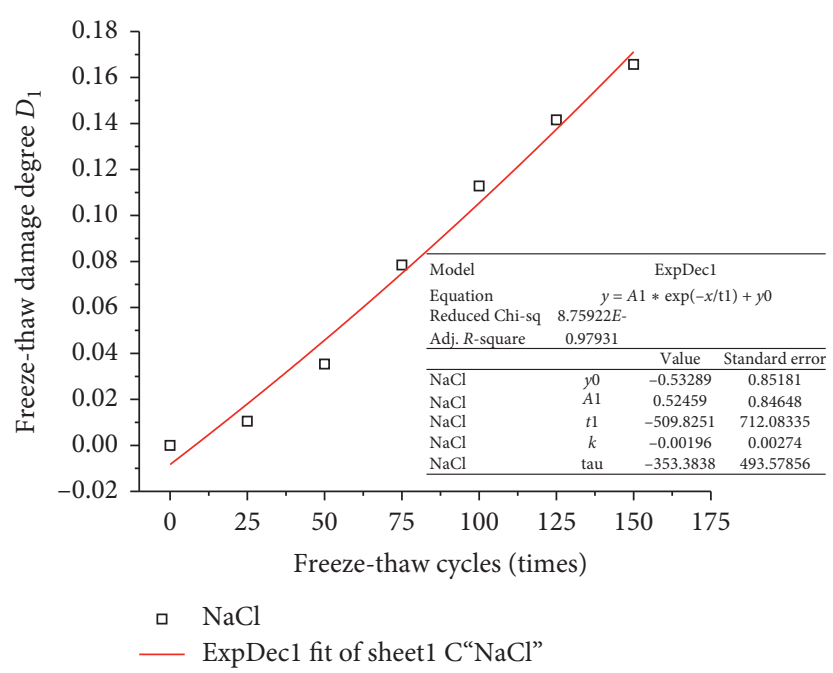

(b)

Figure 12: Fitting curve of freeze-thaw damage $D_{1}$ and freeze-thaw cycles $n$.

where $n$ is the number of freeze-thaw cycles and $N$ is the number of fatigue cycles in the formula.

\subsection{Damage Criterion of Relative Dynamic Elastic Modulus of} Freeze-Thaw Fatigue. As shown in Figure 10, at the end of the second stage of the fatigue test, the dynamic elastic modulus decreases to a stable critical value, which is about $50-70 \%$ of the initial dynamic elastic modulus $E_{d 0}$. When the relative dynamic modulus of elasticity is lower than this value, its descending rate will increase sharply and reach the third stage soon. The third stage accounts for the shorter total fatigue life, and the relative dynamic elastic modulus after the third stage at the end of the trabecular fracture is more discrete. The limit value of the relative dynamic elastic modulus at the end of the second stage is relatively stable, and the first two stages account for more than $90 \%$ of the fatigue life. Therefore, the limit value of the relative dynamic elastic modulus at the end of the second stage can be used as the fatigue failure criterion; that is, when the relative dynamic elastic modulus drops to this limit value, the fatigue failure occurs. Table 7 shows the limit value of the relative dynamic elastic modulus and damage $D$ of specimens after freeze-thaw and fatigue damage.

Table 7 shows that the relative dynamic elastic modulus of water freeze-thaw specimens decreases faster than that of salt-frozen specimens, and the relative dynamic elastic modulus of water-frozen specimens decreases faster than that of salt-frozen specimens. The relative dynamic elastic modulus of water-frozen specimens decreases faster than that of salt-frozen specimens during the freeze-thaw period. The law is not obvious. The damage limit $\mathrm{D}$ obtained by combining the freeze-thaw and fatigue damage can be used as the criterion of fatigue damage of reinforced concrete under the second-order cyclic loading of freeze-thaw damage and fatigue damage. This damage criterion is mainly applicable to reinforced concrete members subjected to the combined action of freeze-thaw and fatigue. The initial dynamic elastic modulus is measured before freeze-thaw failure. After that, the members are subjected to freeze-thaw damage and fatigue load. The dynamic elastic modulus of the members decreases. When the damage limit value $\mathrm{D}$ is reached, the members can be considered to have fatigue failure.

4.3. Fatigue Cumulative Damage Model Based on Residual Deflection. The residual deflection varies with the fatigue test. This change is not a leap but a certain continuity. A continuous function of residual deflection is given, assuming that the growth rate of residual deflection is a constant; i.e., the growth of residual deflection within a unit fatigue number is in constant proportion to the residual deflection that has been generated.

Remember that the residual deflection is $f_{r}(N)$ after $N$ times of cycles. The function is continuous and differentiable. In addition, the fatigue number at the beginning of the fatigue test is set at 0 , the residual deflection at this time is $f_{r 0}$, and the growth rate of residual deflection is $\lambda$, the ratio coefficient between the increment of $\lambda$ unit time $f_{r}(N)$ and $f_{r}$ $(N)$. If $\lambda$ is constant, the increment of the residual deflection of $N$ and $N+\Delta N$ is

$$
f_{r}(N+\Delta N)-f_{r}(N)=\lambda f_{r}(N) \Delta N
$$

That is,

$$
\frac{d f_{r}}{d N}=\lambda f_{r}(N)
$$

The integral of formula (9) can be obtained:

$$
f_{r}(N)=f_{r 0} e^{\lambda N} \text {. }
$$

Formula (10) shows that when $\lambda>0$, the residual deflection increases indefinitely according to the exponential law. However, in the actual test, the growth of residual deflection is mainly divided into three stages. Because the test data are often catastrophic in the later stage of the test, 
TABle 7: Damage criterion of the relative elastic modulus of the test beam after freezing and fatigue.

\begin{tabular}{|c|c|c|c|c|c|c|}
\hline $\begin{array}{l}\text { Freeze- } \\
\text { thaw } \\
\text { medium }\end{array}$ & $\begin{array}{c}\text { Stress } \\
\text { level }\end{array}$ & $\begin{array}{l}\text { Freezing } \\
\text { thawing } \\
\text { times }\end{array}$ & $\begin{array}{c}\text { Relative dynamic elastic } \\
\text { modulus after freezing and } \\
\text { thawing }\end{array}$ & $\begin{array}{l}\text { Relative dynamic elastic } \\
\text { modulus of fatigue at the } \\
\text { second stage }\end{array}$ & $\begin{array}{l}\text { Relative dynamic elastic } \\
\text { modulus at the end of stage } \\
\text { two of freezing- } \\
\text { thawing + fatigue coupling }\end{array}$ & $\begin{array}{c}D \text { limit } \\
\text { value }\end{array}$ \\
\hline \multirow{4}{*}{ Water } & \multirow{8}{*}{0.65} & 0 & 1.000 & 0.60 & 0.600 & 0.400 \\
\hline & & 50 & 0.975 & 0.61 & 0.595 & 0.405 \\
\hline & & 100 & 0.905 & 0.57 & 0.516 & 0.484 \\
\hline & & 150 & 0.819 & 0.58 & 0.475 & 0.525 \\
\hline \multirow{4}{*}{$\mathrm{NaCl}$} & & 0 & 1.000 & 0.60 & 0.600 & 0.400 \\
\hline & & 50 & 0.969 & 0.63 & 0.610 & 0.390 \\
\hline & & 100 & 0.982 & 0.60 & 0.589 & 0.411 \\
\hline & & 150 & 0.957 & 0.54 & 0.517 & 0.483 \\
\hline
\end{tabular}

that is, the third stage, only the first two stages are studied, before the specimen. The growth rate of residual deflection $\lambda$ is not a constant during the two stages of change. It increases first and then decreases, which conforms to the characteristics of the retardation model.

In order to make the residual deflection function closer to the actual situation, assume the normal distribution as shown in Figure 13, and then when the residual deflection reaches the residual deflection when the specimen is destroyed, the growth rate of the residual deflection is 0 , and the growth rate function of the residual deflection $\lambda\left(f_{r}\right)$ can be expressed as follows:

$$
\lambda\left(f_{r}\right)=\lambda\left(\frac{1-f_{r}}{f_{r m}}\right) .
$$

Plug formula (11) into formula (9):

$$
\frac{d f_{r}}{d N}=\lambda\left(1-\frac{f_{r}}{f_{r m}}\right) f_{r}(N) .
$$

The integral of formula (12) can be obtained:

$$
f_{r}(N)=\frac{f_{r m}}{\left[1+\left(f_{r m} / f_{r 0}-1\right) e^{-\lambda N}\right]} .
$$

It is assumed that the degree of concrete damage after $N$ fatigue tests is expressed in terms of $\rho$. We can obtain

$$
\rho=\frac{f_{r}(N)}{f_{r m}}=\frac{1}{\left[1+\left(f_{r m} / f_{r 0}-1\right) e^{-\lambda N}\right]} .
$$

Because it is impossible for the reinforced concrete members to be a constant fatigue stress under the external load as the fatigue load under test, the frost heave stress generated in the concrete is not a constant cyclic stress when the members are subjected to salt freezing cycle. Therefore, the ratio of deflection is corrected by introducing $k_{\varepsilon}$. As the critical point of concrete failure, using $f_{r m}$ is difficult to determine the specific value in the actual test, so it is modified by $k_{\rho}$. The damage degree of concrete can be expressed as follows:

$$
\rho=\frac{f_{r}(N)}{f_{r m}}=\frac{k_{\rho}}{\left[1+\left(k_{\varepsilon} f_{r m} / f_{r 0}-1\right) e^{-\lambda N}\right]} .
$$

There are parameters $f_{r m} / f_{r 0}$ in the modified damage model, and the corresponding correction coefficients $k_{\varepsilon}$ and

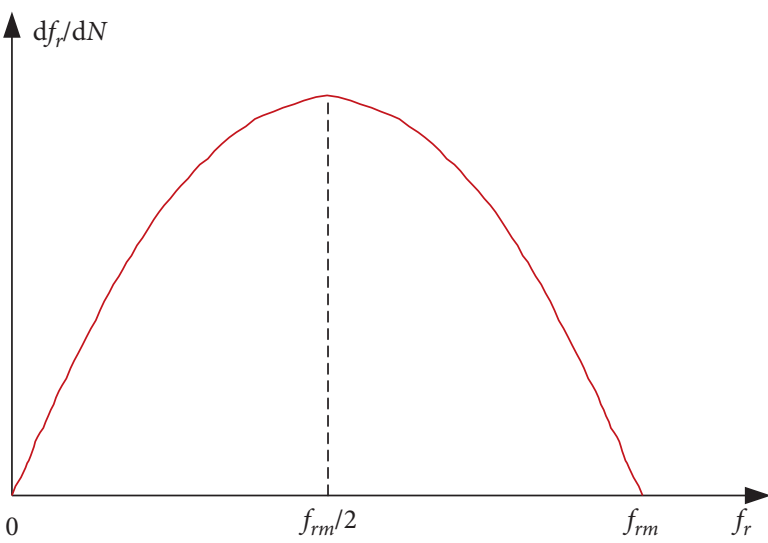

FIGURE 13: Residual deflection rate assumption model.

$k_{\rho}$ and residual deflection change rate $\lambda$ need to be determined. For $f_{r m} / f_{r 0}$ is a parameter related to concrete mix ratio, the fixed concrete mix ratio has specific parameters corresponding to it, and this parameter can be measured by experiment. The ratio $f_{r m} / f_{r 0}$ of the specimens used in this paper is 77.68, and other correction coefficients can be approximated by data fitting.

Because the combination of salt-freeze cycle and fatigue test is closer to the external environment of reinforced concrete specimens, this paper mainly takes the salt-freeze test specimens as an example and uses the damage model established in the previous section to fit the curve of damage degree with the change of salt-freeze cycle and fatigue test times, as shown in Figure 14.

The fitting curve shows that the correlation coefficient $R^{2}$ of damage degree of concrete specimens under salt freezing cycle and fatigue load is above 0.98, which is in good agreement with the experimental data. It shows that the damage model established by residual deflection can accurately reflect the freezing of concrete members under saltfreezing and freezing-thawing damage of external load. It can also be seen from the diagram that the damage degree of concrete specimens tends to increase in the later stage of the fatigue test, but the damage degree of concrete specimens reflected by the damage model in this paper tends to be gentle gradually. Therefore, the error between the test data and the fitting curve mainly comes from the deviation in the later stage of the fatigue test. 


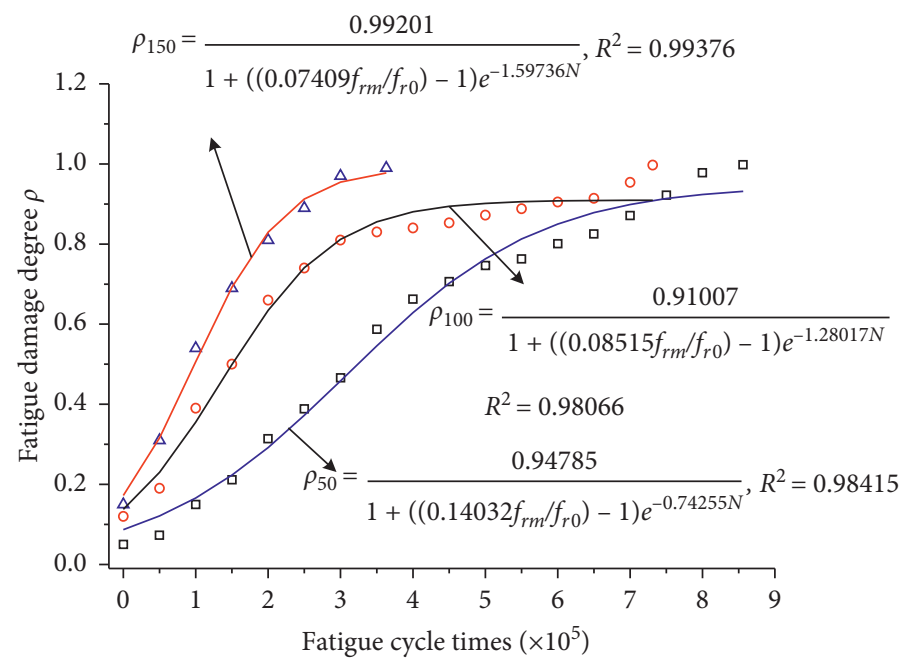

Figure 14: Fitting of damage variable curves of concrete specimens under different salt freezing cycles and fatigue tests.

4.4. Damage Criterion of Fatigue Residual Deflection. The fatigue damage degree $\rho$ represents the ratio of the residual deflection $f_{r}(N)$ of reinforced concrete beams to the critical point $f_{r m}$ of fatigue failure after $N$ cycles of loading. The fatigue damage degree $\rho$ at the end of the second stage is taken as the fatigue failure criterion. The test data are calculated and sorted out, and the fatigue damage degree $\rho$ data at the end of the second stage shown in Table 8 are obtained.

The concrete damage model based on residual deflection combined with the characteristics of concrete material can predict the life of concrete members. The concrete ideas are as follows: (1) Getting the mix ratio of concrete, especially the water binder ratio and air content, which are related to the frost resistance of concrete. (2) Experiments on residual deflection of concrete with different ratios were carried out by enlarging the number of specimens, and the expressions between parameters in the damage model and the ratios of each correction coefficient and concrete were established. (3) The damage model function was set to be 1 , and the fatigue test times under different salt-freeze cycles were calculated, and the corresponding relationship was established. (4) Combining the meteorological conditions, traffic volume, and the corresponding relationship between the axle load of vehicles and (3), the service life of reinforced concrete beam is predicted.

\section{Fatigue Life Prediction Based on Relative Dynamic Modulus of Elasticity}

5.1. Experimental Prediction Formula for Fatigue Life. According to the fatigue test results, the fatigue failure process of the test beam can be divided into three stages. The second stage occupies more than $80 \%$ of the total life. In this paper, only the second stage of fatigue life prediction is considered, and it is considered that the life of the second stage can approximately represent the whole life of the component. The treatment method is more conservative [41], but because of the seriousness of fatigue failure in engineering problems, it needs more conservative design ideas.

In the second stage of the fatigue loading process, the relative dynamic elastic modulus varies linearly with the number of loading cycles $N$. A semiempirical formula for residual fatigue life is derived from formulas (4) and (5):

$$
N=\frac{D_{2}-1+a_{2}}{a_{3}} \text {. }
$$

Formula (16) can be used to predict the ultimate fatigue life of reinforced concrete beams under any constant amplitude cyclic loading. In the actual prediction of fatigue life, only a few load cycles need to be completed. First, the initial dynamic elastic modulus $E_{d 0}$ of fatigue is measured, and then the dynamic elastic modulus $E_{d N}$ is measured when the loading cycle reaches a predetermined number of cycles $N$ and then stops when the relative dynamic elastic modulus drops to about $80 \%$ of the initial value. For the dynamic elastic modulus $a_{2}$ and the slope $a_{3}$, the fatigue life $N$ can be obtained by formula (14), and the residual fatigue life can be obtained by subtracting the number of cycles applied in the test. The advantage of this method is that it is realized by a few fatigue cycles. It is a nondestructive testing method, and the relative dynamic elastic modulus is simple and easy to measure.

5.2. Fatigue Life Prediction Results. The fatigue life of each beam can be predicted by using formula (16) and linear simulation of the second stage relative dynamic elastic modulus. Table 9 lists the predicted and experimental values of fatigue life and calculates the relative error.

It is shown from Table 9 that the predicted fatigue life of reinforced concrete beams is in good agreement with the experimental data for the service condition of formula (16). The average error is $28.2 \%$, and the maximum error is $42.1 \%$. This method only considers the second stage of fatigue failure, so the predicted fatigue life is lower than the actual fatigue life. This prediction method is safer. It can be concluded that the fatigue life of reinforced concrete flexural 
TABLE 8: Fatigue damage degree $\rho$ of the test beam.

\begin{tabular}{lccc}
\hline Freeze-thaw medium & Stress ratio & Freezing and thawing cycles & Fatigue damage degree $\rho$ \\
\hline \multirow{2}{*}{ Water } & & 0 & 0.13 \\
& 0.65 & 50 & 0.10 \\
& & 100 & 0.19 \\
& & 150 & 0.09 \\
$\mathrm{NaCl}$ & \multirow{2}{*}{0.65} & 50 & 0.13 \\
& & 100 & 0.13 \\
& & 150 & 0.39 \\
\hline
\end{tabular}

TABLE 9: Experimental values and predicted values of fatigue life.

\begin{tabular}{|c|c|c|c|c|}
\hline \multirow{2}{*}{ Freeze-thaw medium } & \multirow{2}{*}{ Stress number of freeze-thaw cycles (times) } & \multicolumn{3}{|c|}{ Fatigue life (times) } \\
\hline & & Test value & Predicted value & Relative error (\%) \\
\hline \multirow{4}{*}{ Water } & 0 & 1074282 & 941284 & 12.4 \\
\hline & 50 & 913734 & 575817 & 37.0 \\
\hline & 100 & 559487 & 395593 & 29.3 \\
\hline & 150 & 716758 & 454857 & 36.5 \\
\hline \multirow{3}{*}{$\mathrm{NaCl}$} & 50 & 756219 & 606806 & 19.8 \\
\hline & 100 & 701243 & 557143 & 20.5 \\
\hline & 150 & 493972 & 286156 & 42.1 \\
\hline
\end{tabular}

component can be well predicted by measuring the relative dynamic elastic modulus according to the nondestructive fatigue test data with few cycles.

\section{Conclusion}

(1) After 150 cycles of freeze-thaw cycles, the mass of the reinforced concrete beam increases first and then decreases. The relative dynamic elastic modulus decreases with the freeze-thaw cycles. After 150 freeze-thaw cycles, the relative dynamic elastic modulus of the reinforced concrete specimens in water and $\mathrm{NaCl}$ solution decreased to $67.4 \%$ and $83.4 \%$ of the initial values, respectively.

(2) The fatigue failure of reinforced concrete beams after freeze-thaw is mainly normal section and oblique section failures. Three test beams were damaged by normal cross section, and four test beams were damaged by oblique section. These two kinds of failure are brittle failure. The width of fatigue residual cracks, relative dynamic elastic modulus, and fatigue residual deflection show a rapid development, slow and sustained growth, accelerated destruction stage of three-stage development law with the increase of load cycle times.

(3) The accelerating effect of freeze-thaw on fatigue failure is affected by loading history. Water freezing has a great influence on the initial stage of fatigue load. With the increase of load cycle times, the effect of freeze-thaw is gradually weakened. The acceleration of the salt freezing on the fatigue failure continues to increase with the increase of the number of cycles.

(4) Based on the relative dynamic elastic modulus, a fatigue cumulative damage model after freeze-thaw is established. The fatigue damage criterion of the reinforced concrete beam under the coupling of freeze-thaw and fatigue damage is given, and the fatigue life prediction method based on the relative dynamic elastic modulus is proposed. The average error of the prediction result is $28.2 \%$, and the predicted fatigue life is less than the actual fatigue life. The fatigue life prediction method is conservative and safe.

(5) Taking the maximum fatigue residual deflection of the test beam midspan as the damage factor, a fatigue cumulative damage model based on the fatigue residual deflection is established, and the test data well fitted with the damage model. The residual deflection cumulative damage failure criterion and the residual deflection critical value at the time of fatigue failure are obtained from the fatigue cumulative damage model.

In this paper, aiming at the specific environment of Northeast China in winter, the fatigue cumulative damage model of reinforced concrete beams is established and the fatigue life prediction method based on relative dynamic elastic modulus is proposed, which provides a new method for life prediction of reinforced concrete structures in service. Due to the limitations of test conditions, the model established in this paper has certain limitations. In order to obtain more accurate and applicable prediction model, more experimental beams need to be made for further research.

\section{Data Availability}

The data used to support the findings of the study are included within the article and also in the supplementary materials of the article. 


\section{Conflicts of Interest}

The authors declare no conflicts of interest.

\section{Acknowledgments}

This paper was supported by the Natural Science Foundation Project of Heilongjiang Province (grant number: E201353), Science and Technology Project of Transportation Department of Heilongjiang Province (grant number: 41311603), and Project of Harbin University Young Doctor Research Initiation Fund (grant number: HUDF2019110). The authors wish to express their gratitude to people who helped in carrying out the tests and all those who helped complete and review the manuscript.

\section{Supplementary Materials}

Figures 6 7 and Figures 9 14 in the manuscript of the paper are all generated by the following corresponding table original data. A brief description of the supplementary materials is also in the Figure data.PDF file. (Supplementary Materials)

\section{References}

[1] P. K. Mehta, “Concrete durability: fifty year's progress," in Proceeding of the 2nd International Conference on Concrete Durability, ACI SP126-1, Montreal, Canada, August 1991.

[2] B. Persson, "Internal frost resistance and salt frost scaling of self-compacting concrete," Cement and Concrete Research, vol. 33, no. 3, pp. 373-379, 2003.

[3] A. Cwirzen and V. Penttala, "Aggregate-cement paste transition zone properties affecting the salt-frost damage of highperformance concretes," Cement and Concrete Research, vol. 35, no. 4, pp. 671-679, 2005.

[4] S. Lindmark, Mechanisms of Salt Frost Scaling of Portland Cement Bound Materials: Studies and Hypotheses (TVBM1017), Lund University, Lund, Sweden, 1998.

[5] M. J. Setzer, "Action of forst and de-icing chemicals, basic phenomena and testing," in Proceedings of the RILEM, vol. 30, pp. 2-22, Lyon, France, 1997.

[6] O. Copuroglu, A. Fraaij, and J. Bijen, "Effect of curing conditions on freeze-thaw de-icing salt resistance of blast furnace slag cement mortars," in High Performance Structures and Materials II, pp. 233-241, WIT Press, Ancona, Italy, 2004.

[7] R. D. Hooton, "Performance of HPC made with slag-silica fume ternary cementitious systems exposed to de-icer chloride penetration," in Proceeding of the International Conference on Durability of High-Performance Concrete, Essen, Germany, 2004.

[8] J. Stark and H. M. Ludwig, "Freeze-deicing salt resistance of concrete containing cement rich in slag," in Proceeding Frost Resistance of Concrete, pp. 123-138, E\&FN Spon London, Essen, Germany, 1997.

[9] M. J. Setzer, "The micro ice lens model of frost attack-basics and consequences for testing and application," in Proceedings of the 5th International Symposium on Cement and Concrete, vol. 1, pp. 105-111, Shanghai, China, 2002.

[10] J. Marchand, M. Pigeno, D. Bager, and C Talbot, "Influence of chloride solution concentration on deicer salt scaling deterioration of concrete," ACI Materials Journal, vol. 96, no. 4, pp. 429-435, 1999.
[11] C. Foy, M. Pigeon, and N. Banthia, "Freeze-thaw durability and deicer salt scaling resistance of a 0.25 water-cement ratioconcrete," Cement and Concrete Research, vol. 18, no. 4, pp. 604-614, 1988.

[12] D. J. Janssen and M. B. Snyder, "Mass loss experience with ASTM C 666: with and without deicing salt," in Freeze-Thaw Durability of Concrete, J. Marchand, M. Pigeon, and M. Setzer, pp. 284-295, Chapman \& Hall, London, UK, 1997.

[13] T. Hodgkiess, P. D. Arthur, and J. C. Earl, "Corrosion fatigue of reinforced concrete in seawater," Materials Performance, vol. 23, no. 7, pp. 27-31, 1984.

[14] H. Wang, J. Gong, and Y. Song, "Experimental study on corrosion fatigue of RC beams," China Journal of Building Structures, vol. 25, no. 5, pp. 105-110, 2004.

[15] H. Wang, P. Li, and Z. Xu, "Stiffness analysis and calculation of corroded reinforced concrete beams after fatigue," China Journal of Shandong University of Science and Technology, vol. 12, pp. 44-49, 2010.

[16] R. Dineshkumar and S. Ramkumar, "Review paper on fatigue behavior of reinforced concrete beams," Material Today: Proceedings, 2019.

[17] T. S. Chang and C. E. Kesler, "Fatigue behavior of reinforced concrete beams," Journal Proceedings, vol. 55, no. 8, pp. 245-254, 1958.

[18] P. Stroeven, "Low-cycle compression fatigue of reinforced concrete structures," Procedia Engineering, vol. 2, no. 1, pp. 309-314, 2010.

[19] H.-B. Zhu, Y.-Q. Xu, X. Li, and Z.-W. Yu, "Fatigue behavior of reinforced concrete T-beam," Journal of Highway and Transportation Research and Development (English Edition), vol. 8, no. 3, pp. 46-51, 2014.

[20] B. Liu, L. Tong, and X.-L. Zhao, "Fatigue failure characteristics of steel reinforced concrete girders," Procedia Materials Science, vol. 3, pp. 1717-1722, 2014.

[21] W. Sun, J. Jiang, and J. Wang, "Study on chloride diffusion resistance of HPC and HPFRCC under bending fatigue loading," Materials China, vol. 28, no. 11, pp. 19-25+53, 2009.

[22] M. Oyado, M. Hasegawa, and T. Sato, "Characteristics of fatigue and evaluation of RC beam damaged by accelerated corrosion," Quarterly Report of RTRI, vol. 44, no. 2, pp. 72-77, 2003.

[23] W. Yi and X. Sun, "Experimental investigation on the fatigue behavior of corroded RC beams," China Civil Engineering Journal, vol. 40, no. 3, pp. 6-10, 2007.

[24] J. Wu, C. Wang, and Xu Jia, "Experimental study on flexural behavior of corroded reinforced concrete beams under fatigue loading," China Civil Engineering Journal, vol. 45, no. 10, pp. 118-122, 2012.

[25] S. Li, W. Zhang, X. Gu et al., "Experimental study on fatigue properties of corrosion-accelerated steel bars," Journal of the China Railway Society, vol. 32, no. 5, pp. 93-97, 2010.

[26] Y. Cheng, L. Liao, and B. Hou, Fatigue Strength, pp. 120-125, China Railway Publishing House, Beijing, China, 1990.

[27] M. A. Miner, "Cumulative damage in fatigue," Journal of Applied Mechanics, vol. 12, pp. 159-164, 1945.

[28] J. O. Holman, "Fatigue of concrete by constant and variable amplitude loading," in Fatigue Strength of Concrete Structures, pp. 71-110, ACI, Naples, FL, USA, 1982.

[29] S. S. Manson, Application of a Double Linear Damage Rule to Cumulative Fatigue, ASTM, West Conshohocken, PA, USA, 1967.

[30] A. Buch, Fatigue Strength Calculation, Trans. Tech.Publications, Zurich, Switzerland, 1988. 
[31] S. M. Marco and W. J. Starkey, "A concept of fatigue damage," Transactions of ASME, vol. 76, pp. 627-632, 1954.

[32] D. L. Henry, "A theory of fatigue damage accumulation in steel," Transactions of ASME, vol. 77, pp. 913-918, 1955.

[33] P. Neumann, Fatirue, R. W. Chan et al., Physical Metallurgy, Elsevier Science Publishers, Amsterdam, Netherlands, 3rd edition, 1983.

[34] H. O. Fuchs and R. I. Stephens, Metal Fatigue in Engineering, John Wiley \& Sons, New York, NY, USA, 1980.

[35] J. Lemaitre, J. Ni, and C. Tao, Damage Mechanics Course, Science Publishers, Beijing, China, 1996.

[36] B. Hwanoh, "Cumulative damage theory of concrete under variable-amplitude fatigue loading," ACI Materials Journal, vol. 88, no. 1, pp. 41-48, 1991.

[37] E. Castillo and A. Fernández-Canteli, "A general regression model for lifetime evaluation and prediction," International Journal of Fracture, vol. 107, no. 2, pp. 117-137, 2001.

[38] M. Yu and J. Zhao, "Experimental study on fatigue strength of concrete under complex stress," China Journal of Southwest Jiaotong University, vol. 3, pp. 89-92, 1998.

[39] Chinese Standard (GB/T/50082-2009), Standard for Test Methods of Long-Term Performance and Durability of Ordinary Concrete, Chinese Standard, Beijing, China, 2009.

[40] Chinese Standard (GB/T/50082-2009), Standard for Test Methods of Concrete Structure, pp. 26-33, Chinese Standard, Beijing, China, 2012.

[41] Y. Ma, Y. Xiang, L. Wang, J. Zhang, and Y. Zhang, "Fatigue life prediction for aging RC beams considering corrosive environments," Engineering Structures, vol. 79, pp. 211-221, 2014. 


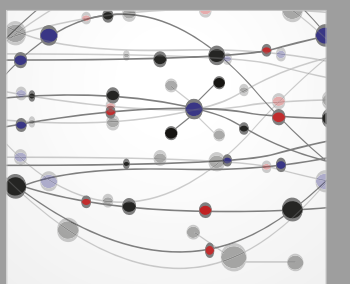

The Scientific World Journal
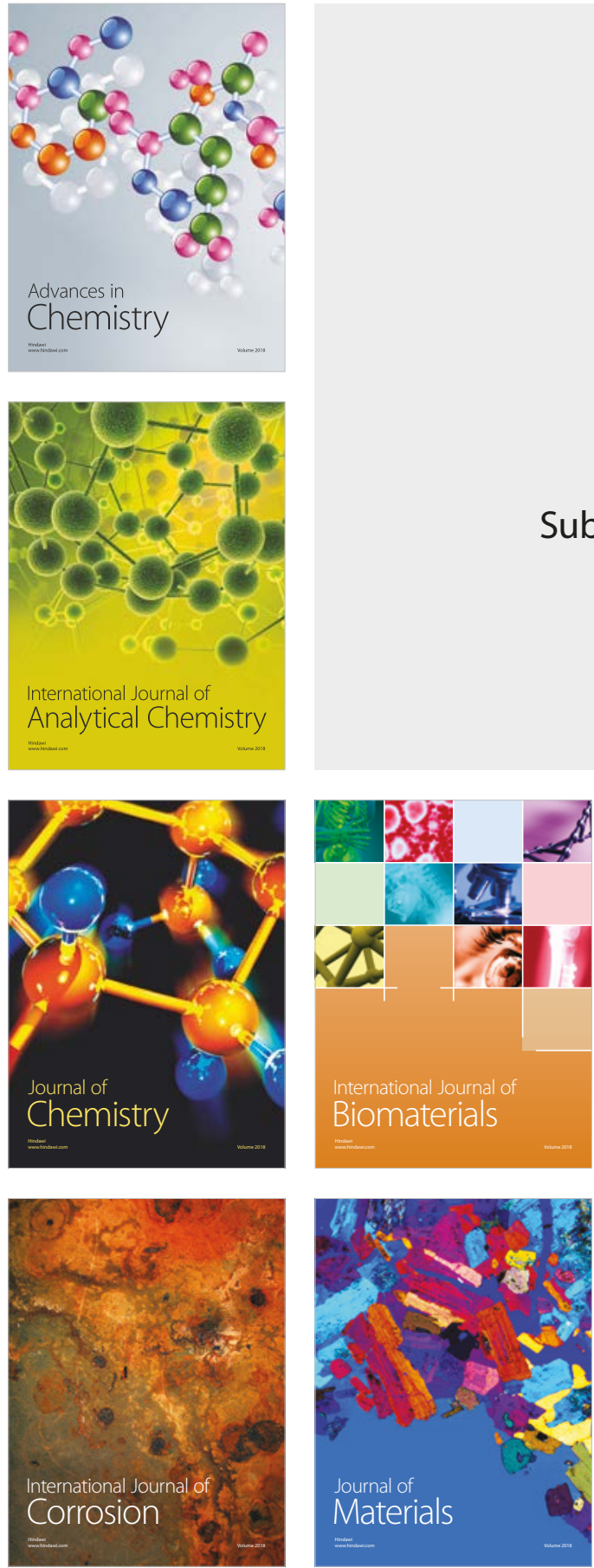

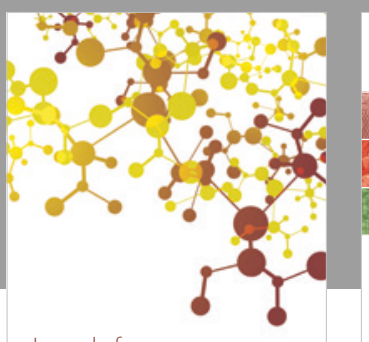

Journal of

Applied Chemistry
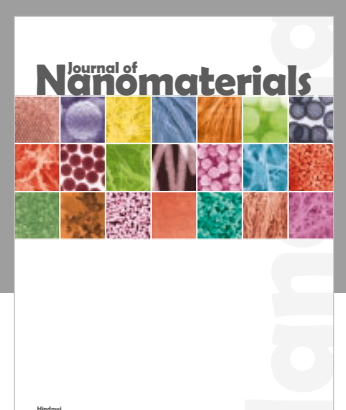

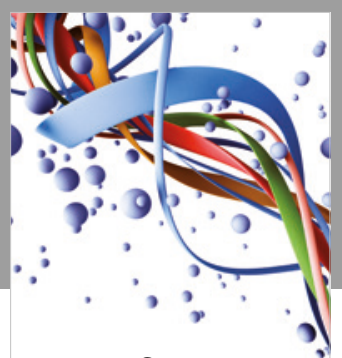

Scientifica

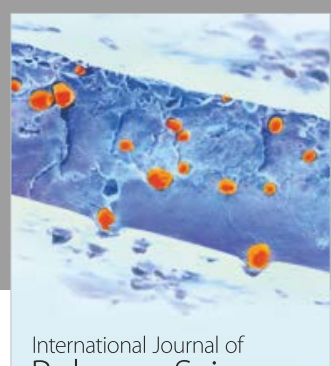

Polymer Science

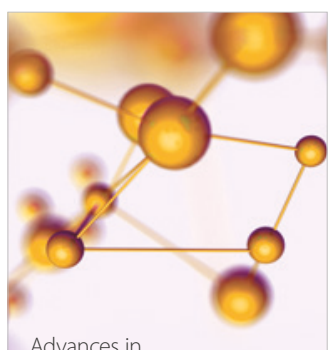

Physical Chemistry
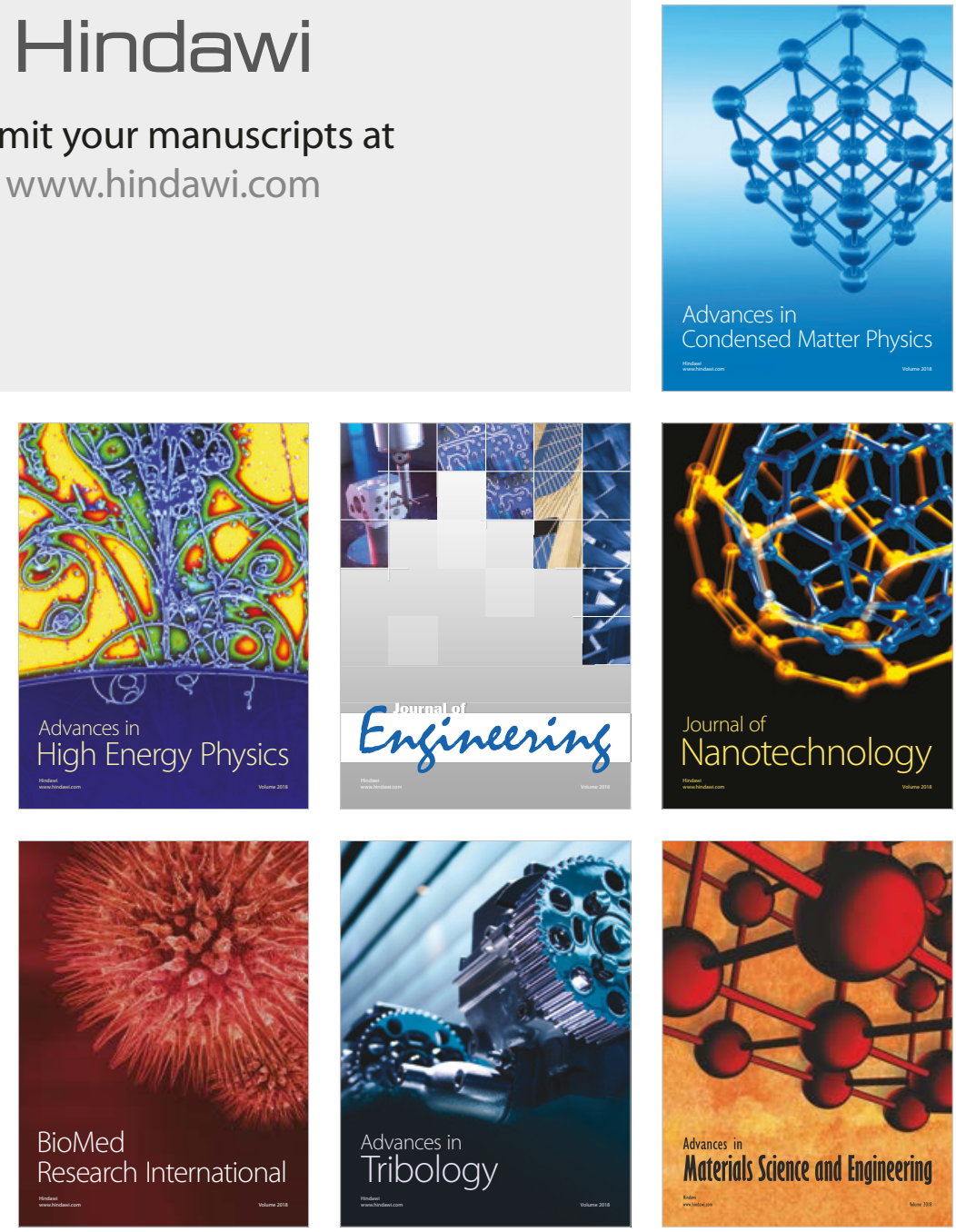